\title{
Hereditary spastic paraplegia type 35 caused by a novel FA2H mutation
}

\author{
Gonca Bektaş${ }^{1}$, Gözde Yeşil², Edibe Pembegül Yıldız¹, Nur Aydınlı1 , Mine Çalışkan¹, \\ Meral Özmen ${ }^{1}$ \\ ${ }^{1}$ Division of Pediatric Neurology, Department of Pediatrics, Istanbul University, Istanbul Faculty of Medicine, ${ }^{2}$ Department \\ of Medical Genetics, Bezmi Alem Vakıf University Faculty of Medicine, Istanbul, Turkey. E-mail: goncabektas@gmail.com \\ Received: 28th November 2016, Revised: 6th February 2017, Accepted: 6th February 2017
}

\begin{abstract}
SUMMARY: Bektaş G, Yeşil G, Yıldız EP, Aydınlı N, Çalışkan M, Özmen M. Hereditary spastic paraplegia type 35 caused by a novel FA2H mutation. Turk J Pediatr 2017; 59: 329-334.

Hereditary spastic paraplegia type 35 (SPG35) is a rare disorder characterized by progressive spasticity. Mutations in the fatty acid 2-hydroxylase $(F A 2 H)$ gene in different loci are responsible for phenotypic variability. We aimed to define the phenotype of SPG35 linked to a novel homozygous mutation c.160_169dup (p.Asp57Glyfs*48) in the $F A 2 H$ gene, and compared with the clinical characteristics and neuroimaging findings of the patients with mutation in the $F A 2 H$ gene. We describe a 5 -year-old boy presenting with spastic paraplegia. He developed a rapid progressive spastic paraplegia and loss of ambulation at an early age, despite the absence of accompanying seizure, neuropathy, cognitive impairment, speech disturbance, and optic atrophy. Neuroimaging revealed white matter changes without brain iron accumulation. A duplication variation; leading to a truncated protein c.160_169dup in the $\mathrm{FA} 2 \mathrm{H}$ gene was found on the homozygous state. A homozygous mutation c.160_169dup in the FA2H gene, which resulted in SPG35 phenotype, may present with rapid progressive spastic paraplegia at an early age.
\end{abstract}

Key words: child, hereditary spastic paraplegia, genetic.

Hereditary spastic paraplegias (HSPs), a heterogeneous group of neurodegenerative disorders, are characterized by progressive spasticity of the lower extremities. HSPs demonstrate variability in terms of age of onset, disease progression rate, and phenotypic features. The clinical features such as, speech and cognitive impairments, optic atrophy, seizures, and neuropathy occurring in HSP overlap with other neurodegenerative disorders. Over 40 different genes with more than 70 loci, which are inherited in autosomal dominant, autosomal recessive or X-linked patterns, have been associated with HSPs.1.

The spectrum of HSPs was expanded by the identification of a mutation in the fatty acid 2-hydroxylase $(F A 2 H)$ gene, which has been recently associated with a novel form of brain iron accumulation ${ }^{2}$. The $F A 2 H$ gene mutation associated with spastic paraplegia has been defined as HSPs type 35 (SPG35) $^{3}$.
Here, we describe the phenotype of SPG35 caused by a novel homozygous mutation c.160_169dup (p.Asp57Glyfs*48) in the FA2H gene, and compare the clinical characteristics and brain imaging findings of the patients with mutations in different loci of the $F A 2 H$ gene.

\section{Case Report}

A 5-year-old boy presented with progressive spastic paraplegia. He was born from healthy consanguineous parents after normal gestation and delivery. Early developmental milestones were normal. He was able to walk at the age of 1 year, and had no history of seizures. At the age of 4 years, he developed a recognizable gait abnormality that had been progressed for 3 months, followed by falling flat on the ground while walking. He had a healthy older sister. Two familial antecedents with similar clinical characteristics were reported to have died aged 15 and 17 years. The genetic data of them is 
unknown. Pedigree is shown in Figure 1.

His anthropometric measurements (weight, height, and head circumference) were placed between 10-25 centile. He had no dysmorphic features. A neurological examination revealed muscle weakness, spasticity, hyperreflexia with ankle clonus in the lower limbs, and positive Babinski sign. The tests of cerebellar function were in normal range. He was unable to walk without support and had urinary incontinence.
The cognitive status according to StanfordBinet Intelligence Scale, and visual examination were within the normal range. Biochemical and metabolic screening (including analysis of plasma lactate, creatine kinase, ammonia, amino acids, very-long-chain fatty acids, and urinary organic acids), electromyography, and motor and sensory nerve conduction studies were unremarkable. Sleep electroencephalography (EEG) showed normal background activity and

(A)

II

III

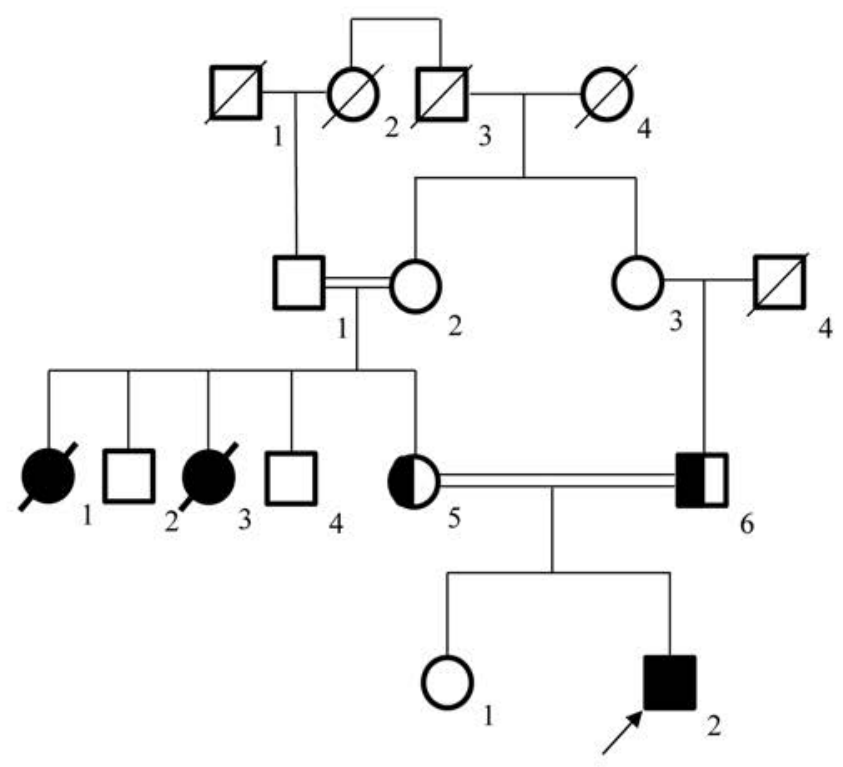

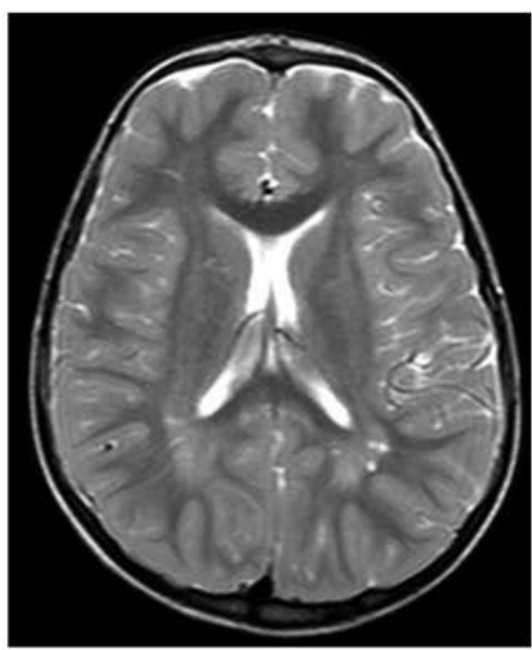

(B)

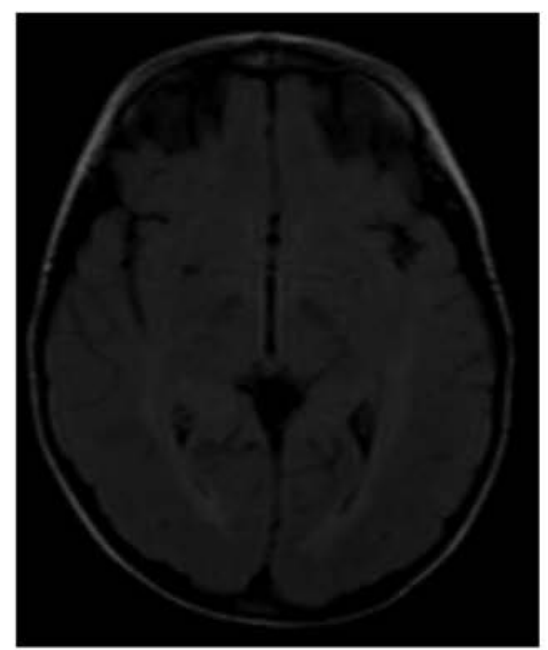

(C)

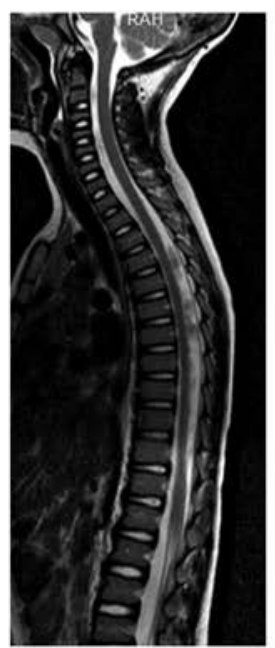

(D)

Fig. 1. (A) Pedigree of the patient with homozygous mutation c.160_169dup (p.Asp57Glyfs*48) in the FA2H gene, (B, C) axial T2-weighted and FLAIR images demonstrates white matter hyperintensities in periventricular region, and (D) spinal magnetic resonance imaging is unremarkable. 
generalized multiple spike discharges. Brain magnetic resonance imaging (MRI) showed white matter hyperintensities in the posterior periventricular areas on T2/FLAIR sequences. The brain iron accumulation was not found. Spinal MRI was normal (Fig. 1).

After obtaining the parents' written informed consent, genomic DNA from peripheral blood was extracted using standard techniques. The samples were enriched for 4813 genes with known associated clinical phenotypes by the TruSight One Sequencing Panel kit on Illumina NextSeq500 platform. Generated variants were reduced by filtering out those with a minor allele frequency greater than $1 \%$ according to various population data. Homozygous rare variants were then filtered due to consanguinity at first step. All candidate variations were evaluated according to clinical inspection. A duplication variation; leading to a truncated protein c.160_169dup (p.Asp57Glyfs*48) in the $\mathrm{FA} 2 \mathrm{H}$ gene was found on the homozygous state, which is caused to a loss of function of the $\mathrm{FA} 2 \mathrm{H}$ gene. Confirmation and segregation analysis of both parents was performed by Sanger sequencing and revealed a heterozygous c.160_169dup (p.Asp57Glyfs*48) change showing their carrier status for the relevant mutation. The carrier status of the sibling was not known. Genetic counseling to the patient and his family were provided.

\section{Discussion}

We described the phenotype of SPG35 caused by a novel homozygous mutation c.160_169dup (p.Asp57Glyfs*48) in the gene encoding $F A 2 H$. The $F A 2 H$ gene encodes an essential protein for the permanence of the myelin sheath around axons ${ }^{3}$. The c.160_169dup (p.Asp57Glyfs*48) duplication variation resulted in a truncated protein in the $F A 2 H$ gene. A compound heterozygous insertion; c.169_170insGCGGGCCAGG (p.Asp57Glyfs*66) leading to a stop codon mutation similar to c.160_169dup (p.Asp57Glyfs*48) was defined in a recent report. The mutation c.169_170insGCGGGCCAGG predicted as deleterious through the results of computational algorithms, indicating that stop codon mutations were critical for the activity of $F A 2 H^{4}$. A homozygous frameshift mutation; c.160_169dup (p.Asp57Glyfs*48) in the $F A 2 H$ gene has been identified for the first time. Other mutations in the $F A 2 H$ gene with distinct clinical manifestations are listed in Table I.

The differential diagnosis of progressive spastic paraplegia; adrenomyeloneuropathy, abetalipoproteinemia, mitochondrial disorders, hereditary motor-sensory neuropathy, and spinal cord lesions, were ruled out in line with laboratory and neuroimaging findings. The phenotype of SPG35 shows variability depending on mutated gene. Early age of onset following a normal development and gait disturbance are common features in SPG35. In our patient, the initial symptoms developed at the 4 years of age. The age of onset was around 4 years in the literature, while there are a few reports indicating a late onset at adulthood $^{1,9}$. Our patient developed spastic paraplegia at an early age of onset as compared with the patient with compound heterozygous mutation c.169_170ins(p.Asp57Glyfs*66) and c.117C $>\mathrm{A}$, and became non-ambulatory at 5 years ${ }^{4}$. The loss of ambulation occurred earlier than the most of the patients with SPG35 caused by $F A 2 H$ mutation. This phenotype of the mutation c.160_169dup (p.Asp57Glyfs*48) demonstrated a rapid progression of spastic paraplegia.

Speech and cognitive impairments, optic atrophy, seizures, and neuropathy have also been described in SPG35 ${ }^{1}$. Speech impairment existed in almost all of the patients. The occurrence of optic atrophy and peripheral neuropathy were not correlated, neuropathy was defined in only two reports 8,13 . The presence of seizure and cognitive decline were also not correlated. Cognitive and speech impairment, optic atrophy, strabismus, seizures, and neuropathy were not yet existent in our case. However, EEG revealed epileptiform discharges indicating the probability of seizure occurrence. While describing the phenotype caused by the mutation c.160_169dup (p.Asp57Glyfs*48), the absence of accompanying features should cautiously be interpreted in view of early age of our patient.

White matter changes; cerebral, cerebellar, brainstem atrophy; and thin corpus callosum were detected in MRI of patients with the FA2H gene mutation. Periventricular white matter changes defined in our patient are 


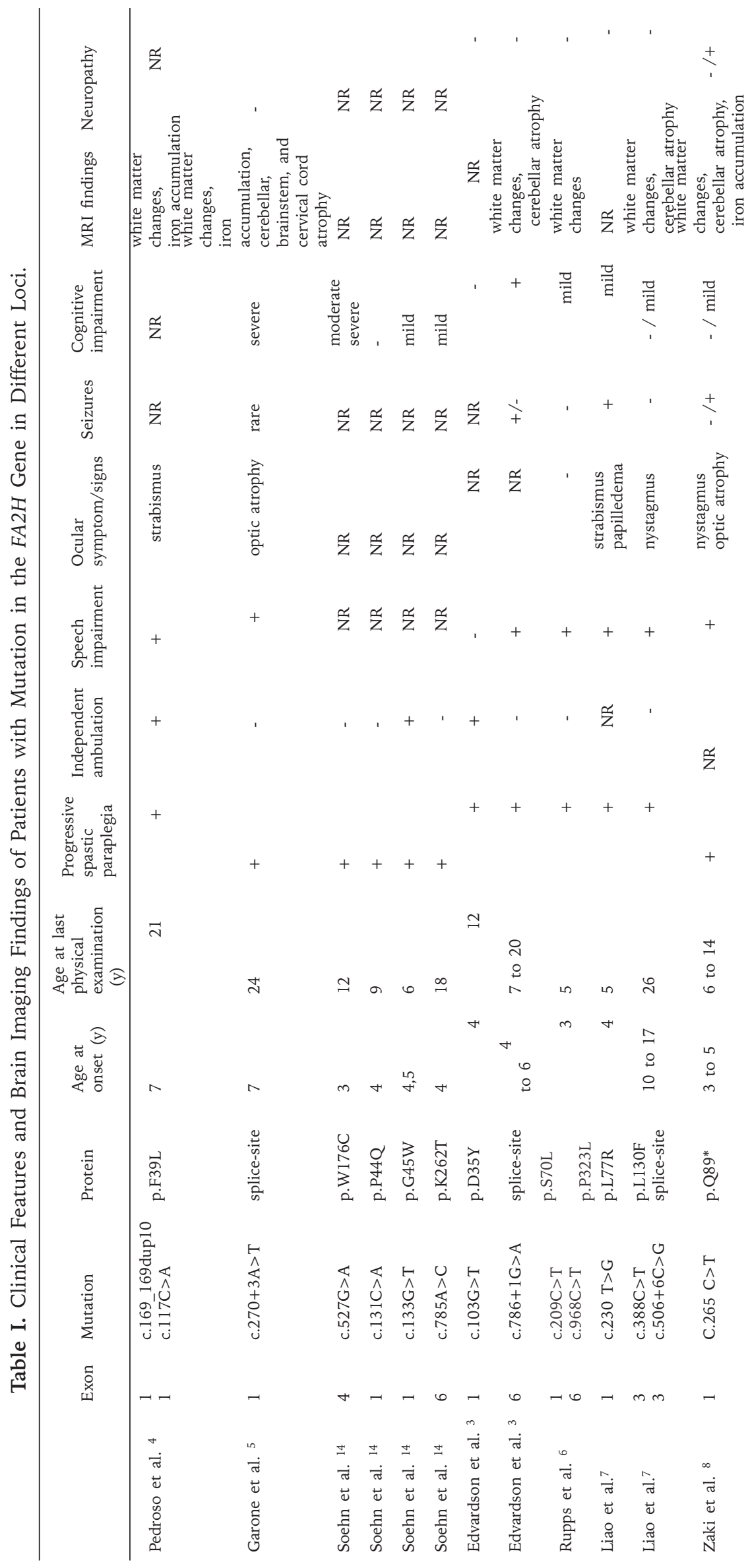




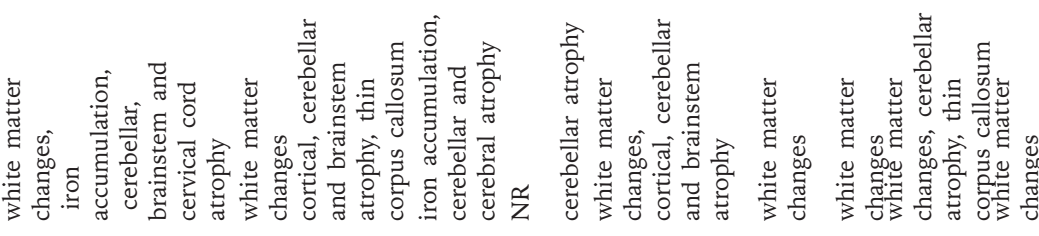

\begin{tabular}{|c|c|c|c|c|c|c|c|c|}
\hline, & $\tilde{z}$ & 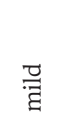 & 号 & + & $\bar{\Xi}$ & 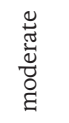 & & \\
\hline+ & \pm & . & $\frac{o}{z} \frac{\tilde{z}}{z}$ & + & + & . & , & . \\
\hline 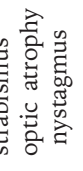 & 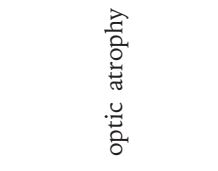 & 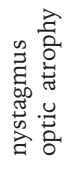 & 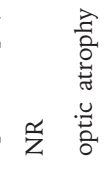 & 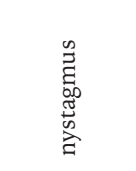 & 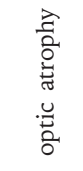 & . & 号 & , \\
\hline+ & + & + & 吕 吕 & + & + & + & + & , \\
\hline , & 吕 & , & 吕 吕 & , & . & , & . & , \\
\hline+ & + & + & $+\quad+$ & + & + & + & + & + \\
\hline $\begin{array}{l}\stackrel{i}{1} \\
\text { D } \\
\text { in }\end{array}$ & 号 & , & 号 高 & $\stackrel{n}{m}$ & $\vec{m}$ & 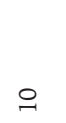 & $\stackrel{m}{\sim}$ & 0 \\
\hline in & $\begin{array}{l}\infty \\
m \\
\vdots\end{array}$ & $\begin{array}{l}q \\
9\end{array}$ & & & & & & \\
\hline$\dot{+}$ & $m$ & $\stackrel{\infty}{m}$ & $\frac{\mathscr{c}}{z}$ m & $r$ & 0 & $n$ & $m$ & + \\
\hline 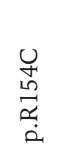 & 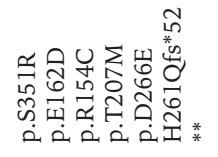 & 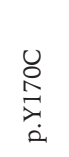 & 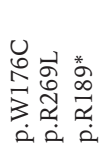 & 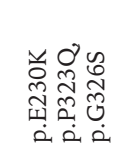 & $\begin{array}{l}u \\
\tilde{\aleph}_{\mathbb{Z}} \\
\stackrel{*}{*}\end{array}$ & 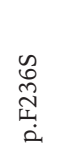 & 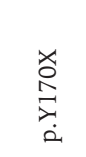 & 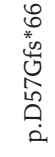 \\
\hline $\begin{array}{l}\hat{A} \\
\hat{O} \\
\text { İ } \\
\text { U. }\end{array}$ & 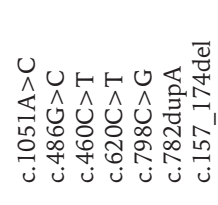 & $\begin{array}{l}0 \\
\hat{1} \\
0 \\
0 \\
0 \\
ن\end{array}$ & 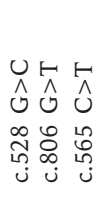 & 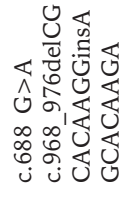 & 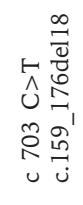 & $\begin{array}{l} \\
\hat{A} \\
\hat{0} \\
0 \\
\stackrel{0}{u}\end{array}$ & 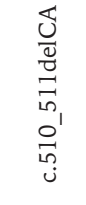 & 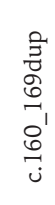 \\
\hline$m$ & Nmmin & n & $\begin{array}{l}+6 \sigma \\
2 \mathbb{N}\end{array}$ & $\ln 6$ & 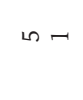 & in & $r$ & - \\
\hline 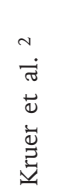 & 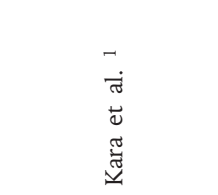 & 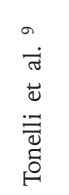 & 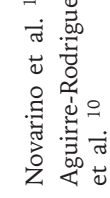 & 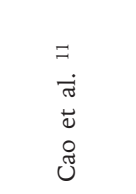 & 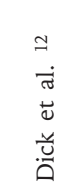 & 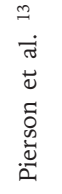 & 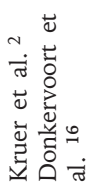 & 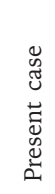 \\
\hline
\end{tabular}


common among SPG351-13. Clinical features and periventricular white matter changes can mimic neurodegenerative disorders such as adrenoleukodystrophy. However, the family history, inheritance pattern, disease course, and negative biochemical and metabolic screening can be indicative of HSPs. Recently, identification of the neurodegeneration with brain iron accumulation expanded the phenotypic spectrum of the disorders associated with the $F A 2 H$ gene mutation $2,4,5,8,9$. Brain iron accumulation was not shown in the patient with homozygous mutation c.160_169dup (p.Asp57Glyfs*48) in the FA2H gene. However, while describing the phenotype caused by the mutation c.160_169dup (p.Asp57Glyfs*48), the absence of accompanying clinical and neuroimaging features should cautiously be interpreted in view of early age of our patient.

In conclusion, the present study identified a novel homozygous mutation c.160_169dup (p.Asp57Glyfs*48) in the FA2H gene resulting in a rapid progressive spastic paraplegia, and an early loss of ambulation.

\section{Acknowledgment}

We kindly thank David Chapman and Cengiz Bektaş for their English language revision.

\section{REFERENCES}

1. Kara E, Tucci A, Manzoni C, et al. Genetic and phenotypic characterization of complex hereditary spastic paraplegia. Brain 2016; 139: 1904-1918.

2. Kruer MC, Paisán-Ruiz C, Boddaert N, et al. Defective $\mathrm{FA} 2 \mathrm{H}$ leads to a novel form of neurodegeneration with brain iron accumulation (NBIA). Ann Neurol 2010; 68: 611-618.

3. Edvardson S, Hama H, Shaag A, et al. Mutations in the fatty acid 2-hydroxylase gene are associated with leukodystrophy with spastic paraparesis and dystonia. Am J Hum Genet 2008; 83: 643-648.

4. Pedroso JL, Handfas BW, Abrahão A, Kok F, Barsottini OG, Oliveira AS. Fatty acid 2-hydroxylase deficiency: clinical features and brain iron accumulation. Neurology 2015; 84: 960-961.

5. Garone C, Pippucci T, Cordelli DM, et al. FA2H-related disorders: a novel c. $270+3 \mathrm{~A}>\mathrm{T}$ splice-site mutation leads to a complex neurodegenerative phenotype. Dev Med Child Neurol 2011; 53: 958-961.

6. Rupps R, Hukin J, Balicki M, Mercimek-Mahmutoglu S, Rolfs A, Dias C. Novel Mutations in FA2H-Associated Neurodegeneration: An Underrecognized Condition? J Child Neurol 2013; 28: 1500-1504.
7. Liao X, Luo Y, Zhan Z, et al. SPG35 contributes to the second common subtype of AR-HSP in China: frequency analysis and functional characterization of FA2H gene mutations. Clin Genet 2015; 87: 85-89.

8. Zaki MS, Selim L, Mansour L, et al. Mutations in FA2H in three Arab families with a clinical spectrum of neurodegeneration and hereditary spastic paraparesis. Clin Genet 2015; 88: 95-97.

9. Tonelli A, D'Angelo MG, Arrigoni F, et al. Atypical adult onset complicated spastic paraparesis with thin corpus callosum in two patients carrying a novel FA2H mutation. Eur J Neurol 2012; 19: e127-e129.

10. Aguirre-Rodríguez FJ, Lucenilla MI, Alvarez-Cubero MJ, Mata C, Entrala-Bernal C, Fernandez-Rosado F. Novel FA2H mutation in a girl with familial spastic paraplegia. J Neurol Sci 2015; 357: 332-334.

11. Cao L, Huang XJ, Chen CJ, Chen SD. A rare family with Hereditary Spastic Paraplegia Type 35 due to novel FA2H mutations: a case report with literature review. J Neurol Sci 2013; 329: 1-5.

12. Dick KJ, Eckhardt M, Paisán-Ruiz C, et al. Mutation of FA2H underlies a complicated form of hereditary spastic paraplegia (SPG35). Hum Mutat 2010; 31: e1251-e1260.

13. Pierson TM1, Simeonov DR, Sincan M, et al. Exome sequencing and SNP analysis detect novel compound heterozygosity in fatty acid hydroxylase-associated neurodegeneration. Eur J Hum Genet 2012; 20: 476479 .

14. Soehn AS, Rattay TW, Beck-Wödl S, Schäferhoff K, Monk D, Döbler-Neumann M, et al. Uniparental disomy of chromosome 16 unmasks recessive mutations of FA2H/SPG35 in 4 families. Neurology 2016; 87: 186191.

15. Novarino G, Fenstermaker AG, Zaki MS, et al. Exome sequencing links corticospinal motor neuron disease to common neurodegenerative disorders. Science 2014; 343: 506-511.

16. Donkervoort S, Dastgir J, Hu Y, et al. Phenotypic variability of a likely $\mathrm{FA} 2 \mathrm{H}$ founder mutation in a family with complicated hereditary spastic paraplegia. Clin Genet 2014; 85: 393-395. 